\title{
Clinical and Radiological Outcomes of Modified Mini-Open and Open Transforaminal Lumbar Interbody Fusion: A Comparative Study
}

\author{
Sudhir Ganesan, Vignesh Jayabalan, Venkatesh Kumar, Karthik Kailash \\ Department of Spine Surgery, Sri Ramachandra Medical University, Chennai, India
}

\begin{abstract}
Study Design: Retrospective cohort study.
Purpose: To compare the clinical and radiological outcomes of modified mini-open transforaminal lumbar interbody fusion (modMOTLIF) and open TLIF (OTLIF).

Overview of Literature: Minimally invasive transforaminal lumbar interbody fusion (MTLIF) is associated with less blood loss, shorter hospital stay, and less pain. However, it has concerns like increased radiation exposure, steep learning curve, and instrumentation cost. We modified the MTLIF technique by direct freehand insertion of pedicle screws using stab incisions without tubular retractors. Methods: The study included 24 patients in the modMOTLIF group and 27 patients in the OTLIF group. The average follow-up period was 25.6 months. Clinical outcomes were measured using Visual Analog Scale (VAS) and Oswestry Disability Index (ODI) scores. Serial $\mathrm{X}$-rays were acquired at $1,3,6,12$, and 24 months to assess the union and presence of instability. We also compared blood loss and length of hospital stay in both groups.

Results: All patients showed progressive improvement in VAS and ODI scores. No differences were observed in the preoperative and postoperative ODI and VAS leg scores between the groups. The immediate postoperative VAS back score was significantly higher in the OTLIF group than in the modMOTLIF group; however, no difference was observed at 1 and 2 years. Radiological analysis showed nonunion in one and two patients in the OTLIF and modMOTLIF groups, respectively. The average blood loss was $63 \mathrm{~mL}$ in the modMOTLIF group and $254 \mathrm{~mL}$ in the OTLIF group. The mean hospital stay was 3 days for the modMOTLIF group and 5 days for the OTLIF group.

Conclusions: modMOTLIF was associated with reduced blood loss and shorter hospital stay compared with OTLIF. No significant differences were observed in the clinical and radiological outcomes between the groups after 2 years despite reduced back pain in the immediate postoperative period in patients who underwent modMOTLIF.
\end{abstract}

Keywords: Modified mini-open transforaminal lumbar interbody fusion; Open transforaminal lumbar interbody fusion

\section{Introduction}

For degenerative lumbar disorders, several surgical techniques have been described after failure of conservative measures. Lumbar interbody techniques have been associated with increased disc space height, which increases the foraminal height, provides adequate decompression of the neural elements, and restores spinal alignment $[1,2]$.

\footnotetext{
Received Aug 7, 2017; Revised Sep 9, 2017; Accepted Oct 1, 2017

Corresponding author: Sudhir Ganesan

Department of Spine Surgery, Sri Ramachandra Medical University, Chennai, Tamil Nadu, India

Tel: +91-9962277126, Fax: +91-4423762467, E-mail: sudhiraxon@gmail.com
} 
Transforaminal lumbar interbody fusion (TLIF) was first described by Harms and Rolinger [3] in 1982, and it has been used widely with good results ever since. However, studies have shown that open TLIF (OTLIF) is associated with extensive soft tissue dissection for attaining a proper lateral to medial trajectory for the pedicle screws, increased blood loss, damage to the paraspinal musculature resulting in increased postoperative pain, and impaired spinal function [4-7]. Mummaneni and Rodts [8] described the technique of mini-open TLIF (MOTLIF) using expandable tubular retractors. Several other authors have described minimally invasive or percutaneous TLIF (pTLIF) [9-15], where they used guide wires and cannulated screws. Both MOTLIF and pTLIF are associated with minimal tissue dissection, less blood loss, shorter hospital stay, reduced postoperative pain, and early mobilization and rehabilitation [10-12]. The concerns with these techniques are increased radiation exposure, steep learning curve, and instrumentation cost [16]. Few authors have reported higher union rates with MOTLIF procedures $[17,18]$. Clinical outcomes have been found to be similar between MOTLIF and OTLIF [19,20]. We modified mini-open TLIF (modMOTLIF) using a single midline incision and special retractors for direct freehand insertion of pedicle screws. The aim of this study was to analyze the clinical and radiological outcomes of MOTLIF and modMOTLIF.

\section{Materials and Methods}

We included 24 patients (average age, 54.63 years) who underwent modMOTLIF and 27 patients (average age,
53.63 years) who underwent OTLIF at Sri Ramachandra Medical University, Chennai between January and July 2014. And the study was performed after obtaining institutional review board approval. Of the 24 patients in the modMOTLIF group, 20 had degenerative listhesis with lumbar canal stenosis and four had lytic listhesis. In the OTLIF group, 21 patients had degenerative listhesis with stenosis and six had lytic listhesis. Patients with dysplastic listhesis and high-grade listhesis (>grade 2) were not included in the study. All patients had a single-level TLIF. L4-L5 TLIF was performed in 14 patients, L3-L4 TLIF in one patient, and L5-S1 TLIF in nine patients in the modMOTLIF group. In the OTLIF group, 15 patients underwent L4-L5 TLIF, one underwent L3-L4 TLIF, and 11 underwent L5-S1 TLIF. The average follow-up period was 25.6 months. Clinical outcomes were measured using the Visual Analog Scale (VAS) and Oswestry Disability Index (ODI) scores. Radiological outcomes were analyzed using serial X-rays at 1, 3, 6, 12, and 24 months. Dynamic $\mathrm{X}$-rays were taken at the end of 1 year to assess the union and presence of instability. We also compared the blood loss and length of hospital stay in both groups.

The collected data were analyzed using IBM SPSS statistics software ver. 23.0 (IBM Corp., Armonk, NY, USA). Descriptive statistics (frequency and percentage analyses) were used for categorical variables, and the mean \pm standard deviation analysis was used for continuous variables. To determine significant differences between the bivariate samples in the independent groups, the unpaired sample $t$-test was used for normal data andthe Mann-Whitney $U$-test for skewed data. The repeated measures analysis of variance was used with
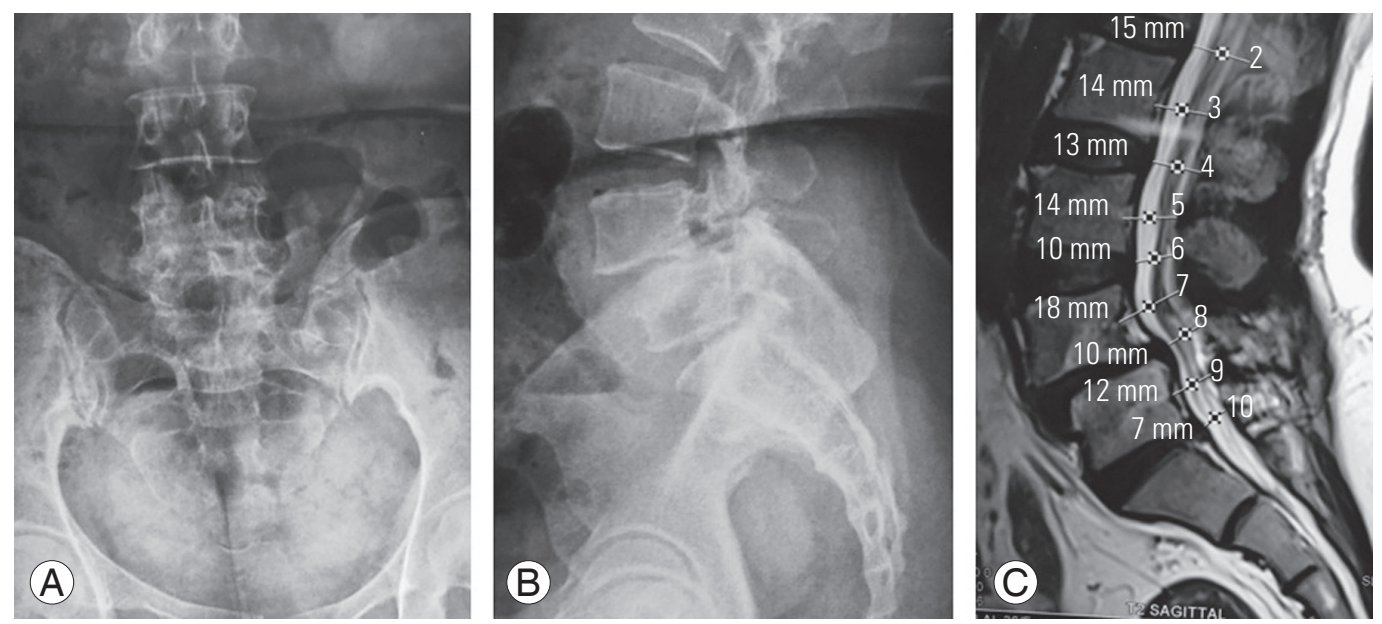

Fig. 1. (A-C) Preoperative $X$-ray and magnetic resonance imaging image of a patient with lytic listhesis 
the Bonferroni test with adjustments for multiple comparisons to control type I errors for normal data; for the skewed data, the Friedman test followed by the Wilcoxon signed rank test was used. To determine the significance of differences in categorical data, the chi-square test was used. For all tests, $p<0.05$ was considered significant.

Patients under general anesthesia were positioned prone over bolsters. A midline skin incision was made from pedicle to pedicle under $\mathrm{C}$-arm guidance. Next, a subdermal dissection was made laterally on both sides by developing a plane between the superficial fascia and the thoracolumbar fascia, which allowed easy retraction. Subdermal dissection stab incisions were made directly over the pedicle under fluoroscopy guidance. The intramuscular plane was established, and special retractors (modified Taylors retractor) were then hooked around the transverse process for direct visualization of the facet joint and the base of the transverse process (Figs. 1, 2). Conventional steps were then followed for freehand insertion of polyaxial pedicle screws into the pedicle without the requirement of a C-arm. Once all the screws were inserted, a $\mathrm{C}$-arm was used to confirm the position of the screws.

On the side of TLIF, screws were inserted into the pedicles as described earlier. The intramuscular plane was established and the facet joint was exposed. The facet joint was knocked off completely under direct vision, and the traversing root and disc space were identified. An incision was made in the disc, which was completely removed, and the end plates were curetted and prepared. Bone grafts were packed anteriorly, followed by a cage packed with grafts. The positions of the screws and cage were confirmed under fluoroscopy, and the wound was closed in layers without a drain.
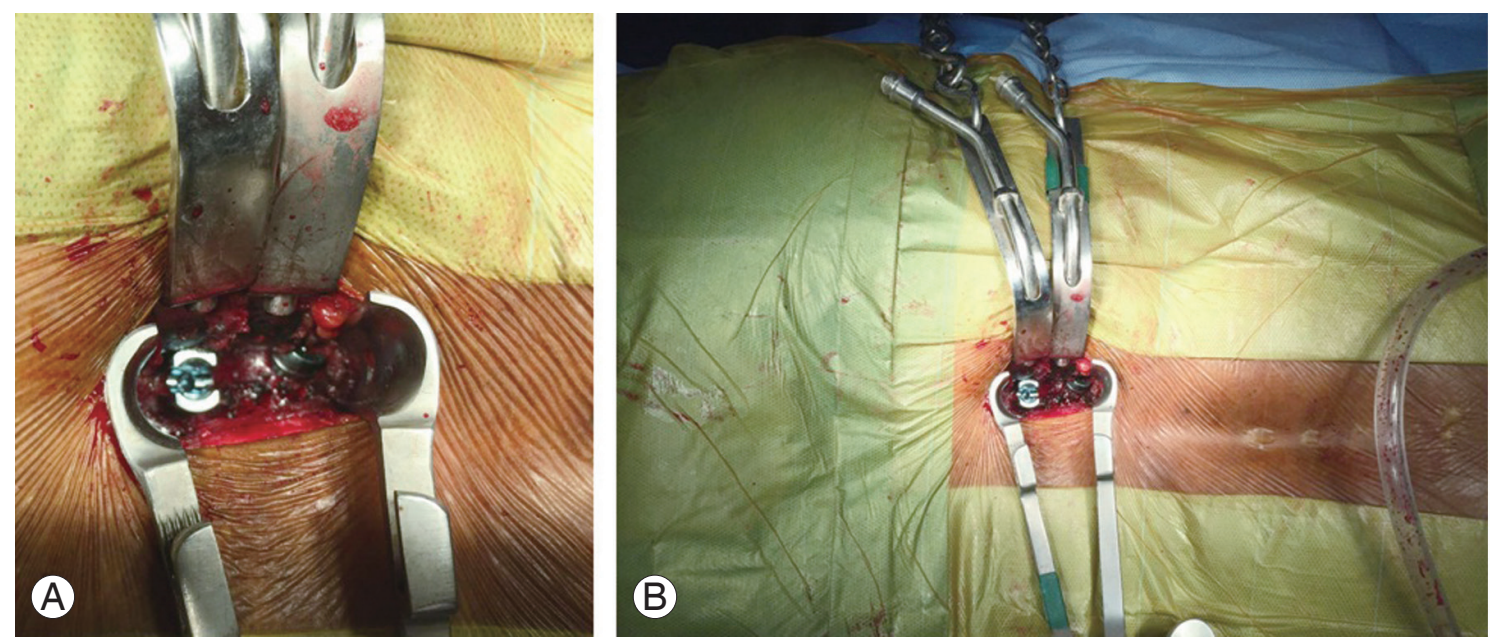

Fig. 2. (A, B) Intraoperative picture of the patient in Fig. 1 showing the special retractor.
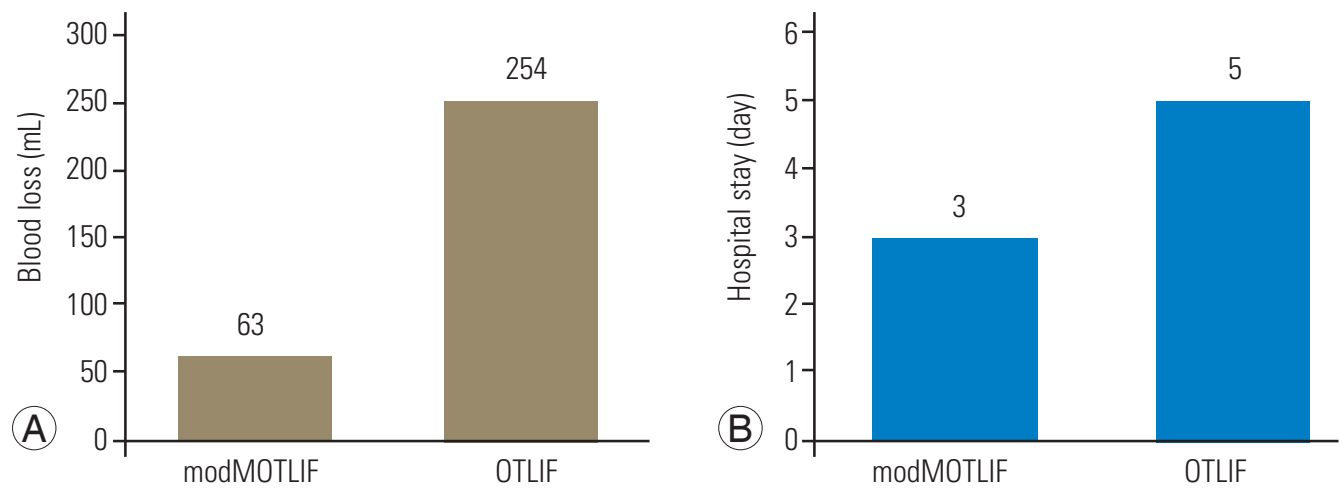

Fig. 3. Bar diagram comparing blood loss (A) and hospital stay (B) of both groups. modMOTLIF, modified mini-open transforaminal interbody fusion; OTLIF, open transforaminal interbody fusion. 
Table 1. Comparison of variables between modMOTLIF and OTLIF

\begin{tabular}{|c|c|c|c|c|c|}
\hline Variable & No. & Mean & Standard deviation & Standard error mean & $p$-value \\
\hline Age (yr) & & & & & 0.605 \\
\hline modMOTLIF & 24 & 54.63 & 7.972 & 1.627 & \\
\hline OTLIF & 27 & 53.63 & 5.617 & 1.081 & \\
\hline Blood loss (mL) & & & & & $0.0005^{*}$ \\
\hline modMOTLIF & 24 & 63 & 21.565 & 4.402 & \\
\hline OTLIF & 27 & 254 & 70.937 & 13.652 & \\
\hline Hospital stay (day) & & & & & $0.0005^{*}$ \\
\hline modMOTLIF & 24 & 3 & 0.676 & 0.138 & \\
\hline OTLIF & 27 & 5 & 1.013 & 0.195 & \\
\hline
\end{tabular}

modMOTLIF, modified mini-open transforaminal interbody fusion; OTLIF, open transforaminal interbody fusion. " $p<0.05$.

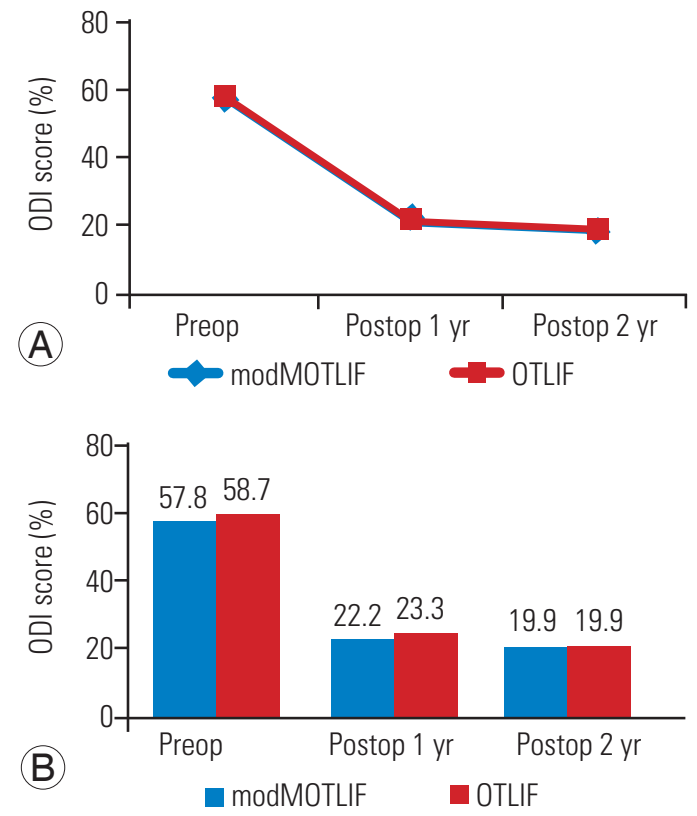

Fig. 4. Bar diagram and chart comparing ODI scores between the groups (preop, postop 1 year, and postop 2 years). modMOTLIF, modified mini-open transforaminal interbody fusion; OTLIF, open transforaminal interbody fusion; ODI, Oswestry Disability Index; Preop, preoperative; Postop, postoperative.

\section{Results}

The mean age of the patients was 54.63 years in the modMOTLIF group and 53.63 years in the OTLIF group. The average blood loss was $63 \mathrm{~mL}$ in the modMOTLIF group and $254 \mathrm{~mL}$ in the OTLIF group; the average hospital stay was 3 days in the modMOTLIF group and 5 days in the OTLIF group (Fig. 3, Table 1). Blood loss and hospital stay were significantly lesser in the modMOTLIF group than in the OTLIF group (Table 1). All 51 patients showed
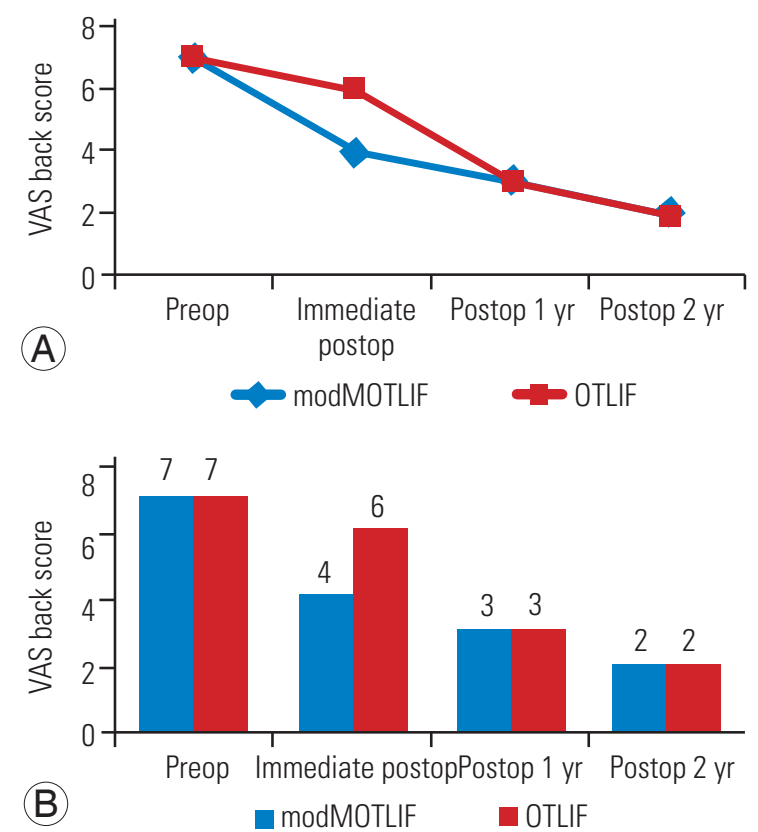

Fig. 5. Bar diagram and chart comparing the VAS back scores between the groups (preop, immediate postop, postop 1 year, and postop 2 years). modMOTLIF, modified mini-open transforaminal interbody fusion; OTLIF, open transforaminal interbody fusion; VAS, Visual Analog Scale; Preop, preoperative; Postop, postoperative.

significant improvements in the VAS and ODI scores. A significant improvement was observed in the VAS back, VAS leg, and ODI scores in both groups $(p<0.05)$ (Figs. $4-6)$. No differences were observed in the preoperative and postoperative ODI scores and VAS leg scores between the groups (Figs. 4-6). The immediate postoperative VAS back score was higher in the OTLIF group than in the modMOTLIF group (Fig. 5). However, the VAS back scores at the end of 1 and 2 years were not significantly 

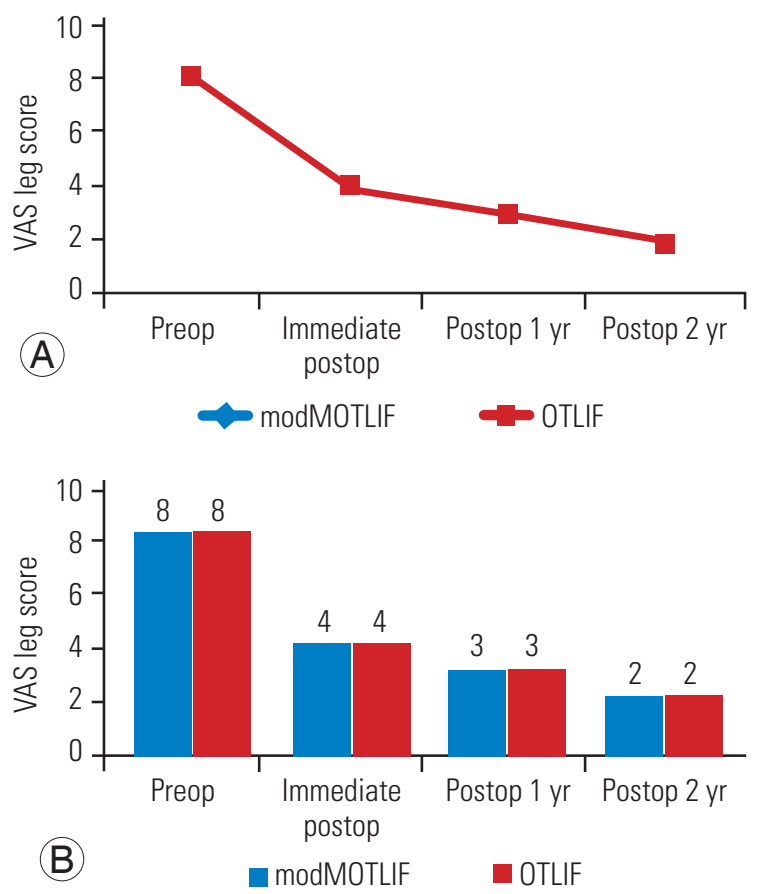

Fig. 6. Bar diagram and chart comparing the VAS leg scores between the groups (preop, immediate postop, postop 1 year, and postop 2 years). modMOTLIF, modified mini-open transforaminal interbody fusion; OTLIF, open transforaminal interbody fusion; VAS, Visual Analog Scale; Preop, preoperative; Postop, postoperative.

different between the two groups (Fig. 5). Radiological analysis showed nonunion in three (5.9\%) out of the 51 patients (two, modMOTLIF group; one, OTLIF group). None of the patients were symptomatic.

\section{Discussion}

Degenerative disc disease is one of the most common disorders affecting the lumbar spine. Surgery is the treatment of choice when conservative treatment fails. Various surgical techniques have been described in the literature. The goal of these surgical techniques is to decompress the neural elements and to achieve fusion between the segments involved. The interbody fusion procedures have several advantages, such as increasing the disc and foramen height, thereby decompressing the nerve roots; achieving $360^{\circ}$ fusion by placing cage and bone grafts anteriorly; and regaining sagittal balance by restoring lumbar lordosis $[1,2]$. TLIF was first described by Harms and Rolinger [3], and since then, it is being used widely with good outcomes. However, the procedure involves extensive paraspinal muscle dissection, which results in muscle denervation, increases postoperative pain, and delays rehabilitation $[4,5]$. It has also been shown that the OTLIF procedure is associated with increased blood loss and increased hospital stay [6,7]. To overcome these disadvantages, minimally invasive techniques such as MOTLIF or pTLIF have been described by various authors with comparable results [8,9-15]. Although the procedures involved have several advantages, there are concerns, such as increased radiation exposure, steep learning curves, and instrumentation cost that have yet to be addressed.

Our technique is a modification of the minimally invasive TLIF procedures, wherein we use a single midline incision from the midpoint of the pedicle to the midpoint of the pedicle below; this gives a cosmetically better scar than that from multiple stab incisions. This incision is followed by subdermal dissection and direct incision over the facet joint between the pedicles. The plane is intramuscular rather than intermuscular as used in the MOTLIF and pTLIF procedures. The attachment of the multifidus muscle is from the spinous process to the superior facet of the vertebra two levels below, whereas the longissimus lumborum is attached to the medial aspect of the transverse process [21]. Because of this arrangement of the muscle fibers, placing a retractor by developing an intermuscular plane leads to the lateral aspect of the facet joint, which requires a more medial angulation for screw placement. After the intramuscular dissection, we place the expandable retractor so that the facet joint is completely exposed. The pedicle screws can be directly inserted freehand with minimal use of fluoroscopy, less angulation, and without the need for guide wires and tubular retractors. Moreover, complete facetectomy is facilitated, and the nerve roots can be thoroughly decompressed under direct vision.

We use an expandable retractor on the side of TLIF and special retractors hung by weights around the transverse process on the contralateral side for placement of screws. The intramuscular approach and the placement of retractors around the transverse process allows direct visualization of the facet joint and the base of the transverse process; therefore, there is no difference between the open technique and our technique. The steep learning curve has been noted as a disadvantage of the MOTLIF and pTLIF procedures by a few authors. Our technique is relatively easy and simple, and surgeons who perform OTLIF will not have any difficulty with this method.

Radiation exposure, which is a major concern for these procedures, is also taken care of by this method. Fluoroscopy in our study was used to check and confirm the 
levels, to mark the pedicles, and finally for ascertaining the position of the screws and cage. Because there are no blind steps involved in this procedure, the fluoroscopy time is significantly reduced.

Rodríguez-Vela et al. [22] showed no significant differences in ODI and VAS back and leg scores in both groups. In our study, there was a significant improvement based on the ODI score in the clinical outcomes in both groups. The two groups had a significant improvement in the postoperative ODI scores at 1 year and 2 years; no differences were observed between the two groups. There were no differences in the preoperative VAS for back pain and leg pain in both groups. The VAS score for back pain in the immediate postoperative period was significantly lesser in the modMOTLIF group than in the OTLIF group. This result could be because of the extensive tissue dissection and muscle injury that occurs during the open procedure, which is significantly less in our technique. However, at 1 and 2 years postoperatively, no differences were observed in the VAS back scores. The postoperative VAS leg pain scores were similar between the groups.

\section{Conclusions}

The modMOTLIF procedure is associated with reduced blood loss and shorter hospital stay compared with the OTLIF procedure. No differences were observed in the clinical and radiological outcomes between the groups after 1 year despite improved short-term clinical outcomes in patients who underwent modMOTLIF.

\section{Conflict of Interest}

No potential conflict of interest relevant to this article was reported.

\section{References}

1. Mehta VA, McGirt MJ, Garces Ambrossi GL, et al. Trans-foraminal versus posterior lumbar interbody fusion: comparison of surgical morbidity. Neurol Res 2011;33:38-42.

2. Moskowitz A. Transforaminal lumbar interbody fusion. Orthop Clin North Am 2002;33:359-66.

3. Harms J, Rolinger H. A one-stager procedure in operative treatment of spondylolistheses: dorsal traction-reposition and anterior fusion (author's transl).
Z Orthop Ihre Grenzgeb 1982;120:343-7.

4. Tsutsumimoto T, Shimogata M, Ohta H, Misawa H. Mini-open versus conventional open posterior lumbar interbody fusion for the treatment of lumbar degenerative spondylolisthesis: comparison of paraspinal muscle damage and slip reduction. Spine (Phila Pa 1976) 2009;34:1923-8.

5. Rodriguez-Vela J, Lobo-Escolar A, Joven-Aliaga E, et al. Perioperative and short-term advantages of miniopen approach for lumbar spinal fusion. Eur Spine J 2009;18:1194-201.

6. Gejo R, Matsui H, Kawaguchi Y, Ishihara H, Tsuji H. Serial changes in trunk muscle performance after posterior lumbar surgery. Spine (Phila Pa 1976) 1999;24:1023-8.

7. Rantanen J, Hurme M, Falck B, et al. The lumbar multifidus muscle five years after surgery for a lumbar intervertebral disc herniation. Spine (Phila Pa 1976) 1993;18:568-74.

8. Mummaneni PV, Rodts GE Jr. The mini-open transforaminal lumbar interbody fusion. Neurosurgery 2005;57(4 Suppl):256-61.

9. Ozgur BM, Yoo K, Rodriguez G, Taylor WR. Minimally-invasive technique for transforaminal lumbar interbody fusion (TLIF). Eur Spine J 2005;14:887-94.

10. Foley KT, Holly LT, Schwender JD. Minimally invasive lumbar fusion. Spine (Phila Pa 1976) 2003;28(15 Suppl):S26-35.

11. Schwender JD, Holly LT, Rouben DP, Foley KT. Minimally invasive transforaminal lumbar interbody fusion (TLIF): technical feasibility and initial results. J Spinal Disord Tech 2005;18 Suppl:S1-6.

12. Holly LT, Schwender JD, Rouben DP, Foley KT. Minimally invasive transforaminal lumbar interbody fusion: indications, technique, and complications. Neurosurg Focus 2006;20:E6.

13. Khoo LT, Palmer S, Laich DT, Fessler RG. Minimally invasive percutaneous posterior lumbar interbody fusion. Neurosurgery 2002;51(5 Suppl):S166-81.

14. Park P, Foley KT. Minimally invasive transforaminal lumbar interbody fusion with reduction of spondylolisthesis: technique and outcomes after a minimum of 2 years' follow-up. Neurosurg Focus 2008;25:E16.

15. Scheufler KM, Dohmen H, Vougioukas VI. Percutaneous transforaminal lumbar interbody fusion for the treatment of degenerative lumbar instability. Neurosurgery 2007;60(4 Suppl 2):203-12. 
16. Wang J, Zhou Y, Zhang ZF, Li CQ, Zheng WJ, Liu J. Comparison of one-level minimally invasive and open transforaminal lumbar interbody fusion in degenerative and isthmic spondylolisthesis grades 1 and 2. Eur Spine J 2010;19:1780-4.

17. Park Y, Ha JW. Comparison of one-level posterior lumbar interbody fusion performed with a minimally invasive approach or a traditional open approach. Spine (Phila Pa 1976) 2007;32:537-43.

18. Logroscino CA, Proietti L, Pola E, Scaramuzzo L, Tamburrelli FC. A minimally invasive posterior lumbar interbody fusion for degenerative lumbar spine instabilities. Eur Spine J 2011;20 Suppl 1:S41-5.

19. Shunwu F, Xing Z, Fengdong Z, Xiangqian F. Minimally invasive transforaminal lumbar interbody fusion for the treatment of degenerative lumbar diseases. Spine (Phila Pa 1976) 2010;35:1615-20.
20. Dhall SS, Wang MY, Mummaneni PV. Clinical and radiographic comparison of mini-open transforaminal lumbar interbody fusion with open transforaminal lumbar interbody fusion in 42 patients with longterm follow-up. J Neurosurg Spine 2008;9:560-5.

21. Hoh DJ, Wang MY, Ritland SL. Anatomic features of the paramedian muscle-splitting approaches to the lumbar spine. Neurosurgery 2010;66(3 Suppl Operative):13-24.

22. Rodriguez-Vela J, Lobo-Escolar A, Joven E, MunozMarin J, Herrera A, Velilla J. Clinical outcomes of minimally invasive versus open approach for onelevel transforaminal lumbar interbody fusion at the 3- to 4-year follow-up. Eur Spine J 2013;22:2857-63. 